\title{
Directed Nano-scale Self-Assembly of Molecular Wires Interconnecting Nodal Points using Monte Carlo Simulations
}

\author{
A. M. Boscoboinik, S. J. Manzi, W. T. Tysoe, V. D. Pereyra, and J. A. Boscoboinik \\ Chem. Mater., Just Accepted Manuscript • DOI: 10.1021/acs.chemmater.5b02413 • Publication Date (Web): 10 Sep 2015
}

Downloaded from http://pubs.acs.org on September 10, 2015

\section{Just Accepted}

"Just Accepted" manuscripts have been peer-reviewed and accepted for publication. They are posted online prior to technical editing, formatting for publication and author proofing. The American Chemical Society provides "Just Accepted" as a free service to the research community to expedite the dissemination of scientific material as soon as possible after acceptance. "Just Accepted" manuscripts appear in full in PDF format accompanied by an HTML abstract. "Just Accepted" manuscripts have been fully peer reviewed, but should not be considered the official version of record. They are accessible to all readers and citable by the Digital Object Identifier (DOI®). "Just Accepted" is an optional service offered to authors. Therefore, the "Just Accepted" Web site may not include all articles that will be published in the journal. After a manuscript is technically edited and formatted, it will be removed from the "Just Accepted" Web site and published as an ASAP article. Note that technical editing may introduce minor changes to the manuscript text and/or graphics which could affect content, and all legal disclaimers and ethical guidelines that apply to the journal pertain. ACS cannot be held responsible for errors or consequences arising from the use of information contained in these "Just Accepted" manuscripts. 


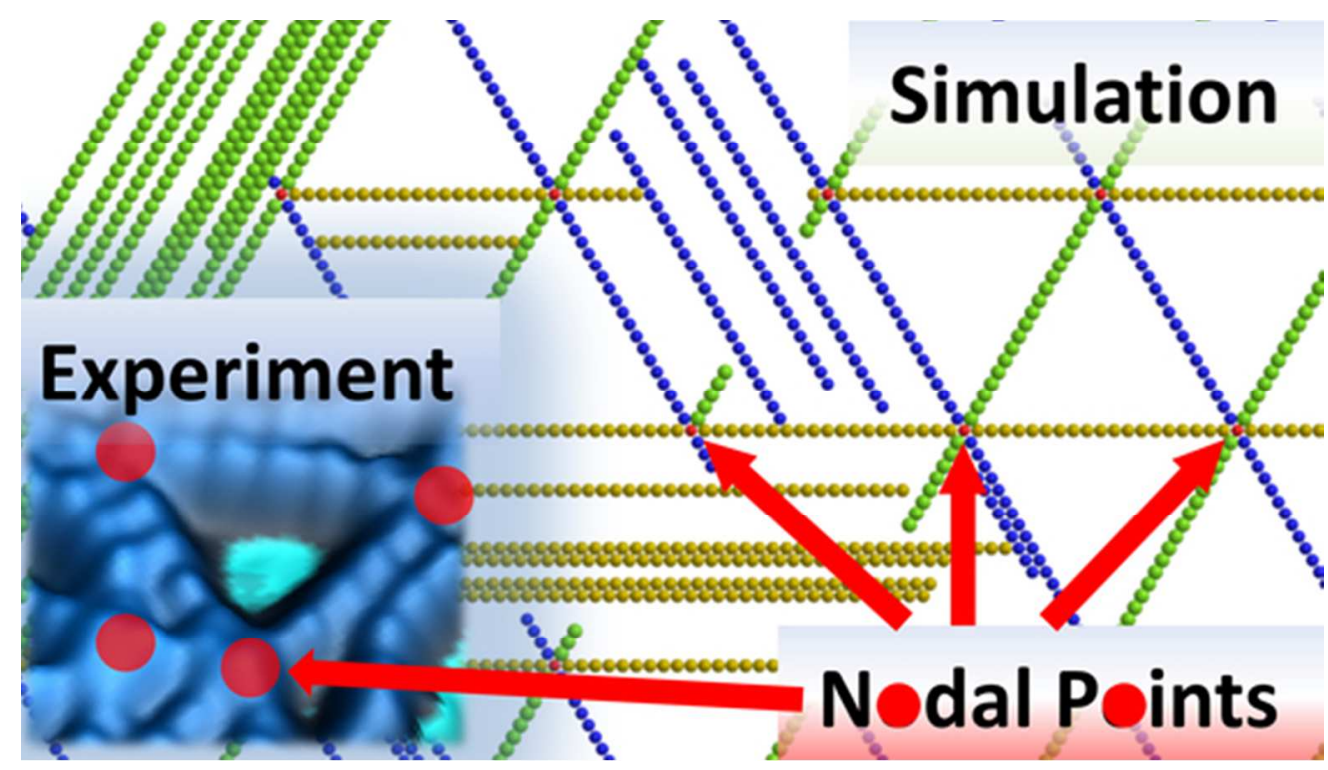

$47 \times 27 \mathrm{~mm}(300 \times 300$ DPI $)$ 


\title{
Directed Nano-scale Self-Assembly of Molecular Wires Interconnecting Nodal Points using Monte Carlo Simulations
}

\author{
A. M. Boscoboinik ${ }^{1}$, S. J. Manzi ${ }^{1}$, W.T.Tysoe ${ }^{2}$, V. D. Pereyra ${ }^{1}$, J. A. Boscoboinik*3 \\ ${ }^{1}$ Instituto de Fisica Aplicada INFAP-CONICET-Departamento de Fisica-Universidad Nacional de San Luis, \\ Chacabuco 917-5700-San Luis, Argentina. \\ ${ }^{2}$ Department of Chemistry and Biochemistry and Laboratory for Surface Studies, University of Wisconsin- \\ Milwaukee, Milwaukee, Wisconsin 53211, United States. \\ ${ }^{3}$ Center for Functional Nanomaterials, Brookhaven National Laboratory, Upton, NY 11973-5000, United \\ States.
}

\section{Abstract}

The influence of directing agents in the self-assembly of molecular wires to produce twodimensional electronic nanoarchitectures is studied here using a Monte Carlo approach to simulate the effect of arbitrarily locating nodal points on a surface, from which the growth of self-assembled molecular wires can be nucleated. This is compared to experimental results reported for the self-assembly of molecular wires when 1,4-phenylenediisocyanide (PDI) is adsorbed on $\mathrm{Au}(111)$. The latter results in the formation of (Au-PDI) $n$ organometallic chains, which were shown to be conductive when linked between gold nanoparticles on an insulating substrate. The present study analyzes, by means of stochastic methods, the influence of variables that affect the growth and design of self-assembled conductive nano-architectures, such as the distance between nodes, coverage of the monomeric units that leads to the formation of the desired architectures and the interaction between the monomeric units. This work proposes an approach and sets the stage for the production of complex 2D nanoarchitectures using a bottom-up strategy but including the use of current state-of-the-art top-down technology as an integral part of the self-assembly strategy.

*Corresponding author: jboscoboinik@bnl.gov (J.A. Boscoboinik) 


\section{Introduction}

Understanding how single molecules self-organize into conductive architectures at the subnanometer scale is crucial for the development of new technologies using the long-overdue "bottom-up" approach. Within the next few years, the limit in the size of objects that can be manipulated (or etched) in a reliable and industrially viable manner will be reached and completely new ways of approaching the fabrication will be required. ${ }^{1}$ This has already been anticipated by Feynman's famous lecture "There is Plenty of Room at the Bottom", ${ }^{2}$ when he proposed the idea of constructing electronic circuits by assembling atoms; a so-called "bottomup" rather than the traditional "top-down" approach. This notion became more concrete with Aviram and Ratner's suggestions for molecular architectures that were proposed to act as diodes. $^{3}$ Subsequently, a large amount of work has been carried out to measure the electrontransport properties of individual molecules, for example using break junctions or scanningprobe microscopy tips ${ }^{4-5}$, providing a better understanding of the charge-transport behavior at the single molecule level.

Naturally, the next natural step is the preparation of these nanoarchitectures. Strategies involving self-assembled monolayers have shown very promising results, such as in the case of Self-Assembled Monolayer Field-Effect Transistors (SAMFETs). ${ }^{6}$ However, the more spatially efficient controlled formation of conductive nanoarchitectures within a single monolayer is a much more challenging problem. While scanning probe techniques such as scanning tunneling microscopy (STM) have shown elegantly how the manipulation of individual atoms and molecules is in principle possible, these methods are mostly of academic interest, ${ }^{7-8}$ and their translation into industrially relevant processes for mass production of nano-electronic devices is questionable and unlikely to be capable of efficiently assembling the large number of devices required to fabricate realistic circuits.

An alternative method is explored here, inspired mostly by complex nanoarchitectures found in nature. This consists of creating devices at the nanoscale by allowing the building blocks to selforganize into the desired structures by tuning the environmental conditions ${ }^{9}$ on an already prepatterned substrate. Establishing how such a targeted self-assembly can be achieved to form a 
specific desired structure is not trivial and requires a detailed understanding of the selfassembly process and, in turn, of the interactions between all the species involved. The nature of these interactions has been explored in innumerable studies of the self-assembly and selforganization processes. ${ }^{10-12}$

In terms of conductive self-assembled structures, it has been shown that bifunctional molecules with ligands that bind strongly to gold, such as isocyanides and thiols, can spontaneously oligomerize on gold surfaces ${ }^{13}$ and are also capable of forming conductive bridges between gold nanoelectrodes on an insulating mica substrate. ${ }^{14}$ The structure of organometallic wires made from 1,4-phenylenediisocyanide (PDI) and Au adatoms consisting of (-Au-PDI-) n chains was previously imaged by STM down to the atomic scale, where it could be seen that the distance between individual monomeric (Au-PDI-) units is $1.1 \mathrm{~nm} .{ }^{13,15}$ This type of self-assembly chemistry offers the possibility of interconnecting between gold nanoelectrodes. Furthermore, based on an analysis of the self-assembly mechanism, ${ }^{16}$ it was suggested that dipolar analogs of these molecules could be aligned by the imposition of an external electric field between nanoelectrodes.

An interesting twist in the approach proposed here to the self-assembly of circuits is to make use of current top-down nanofabrication techniques, which allow the preparation of features with sizes in the order of $10 \mathrm{~nm}$, to produce judiciously tuned nanoelectrodes. These will act as guiding and anchoring points for the directed self-organization of interconnections between them, leading to fabricating devices with complex electronic and structural functions.

In the work described here, and based on the linear self-assembly mechanism discussed above, a simplified model is explored using Monte Carlo simulations and the lattice gas model in order to examine the outcome structures as a function of various parameters that influence the evolution of the nano-architecture. These parameters are, (a) the interaction energy " $w$ " between the entities involved in the process, (b) the coverage $\theta$ of monomeric units, i.e.: the "building blocks", (c) distance " $m$ " between nucleation sites, (d) temperature and (e) time elapsed from the deposition of the precursor molecules on the surface. Understanding the influence of these parameters in the resulting structure will aid the future design of nano- 
architectures produced by directed self-assembly. Note that we have chosen to use the stochastic approach provided by Monte Carlo simulations to address the self-assembly, to mimic the way nature carries out this process.

\section{Theoretical Model}

The model proposed here is inspired by the experimental system of (PDI-Au $n$ on $A u(111)$. However, while we aim to capture some of the fundamental features of the experimental system, the main purpose of this work is to set the stage for the methodologies to address the process of directed self-assembly in general. In order to do so in a simple and clear way we have imposed restrictions to the model that, to some extent, differ from the experimental system. Nevertheless, as will be shown from the results, many of the features arising from the simulation do indeed reflect those seen experimentally.

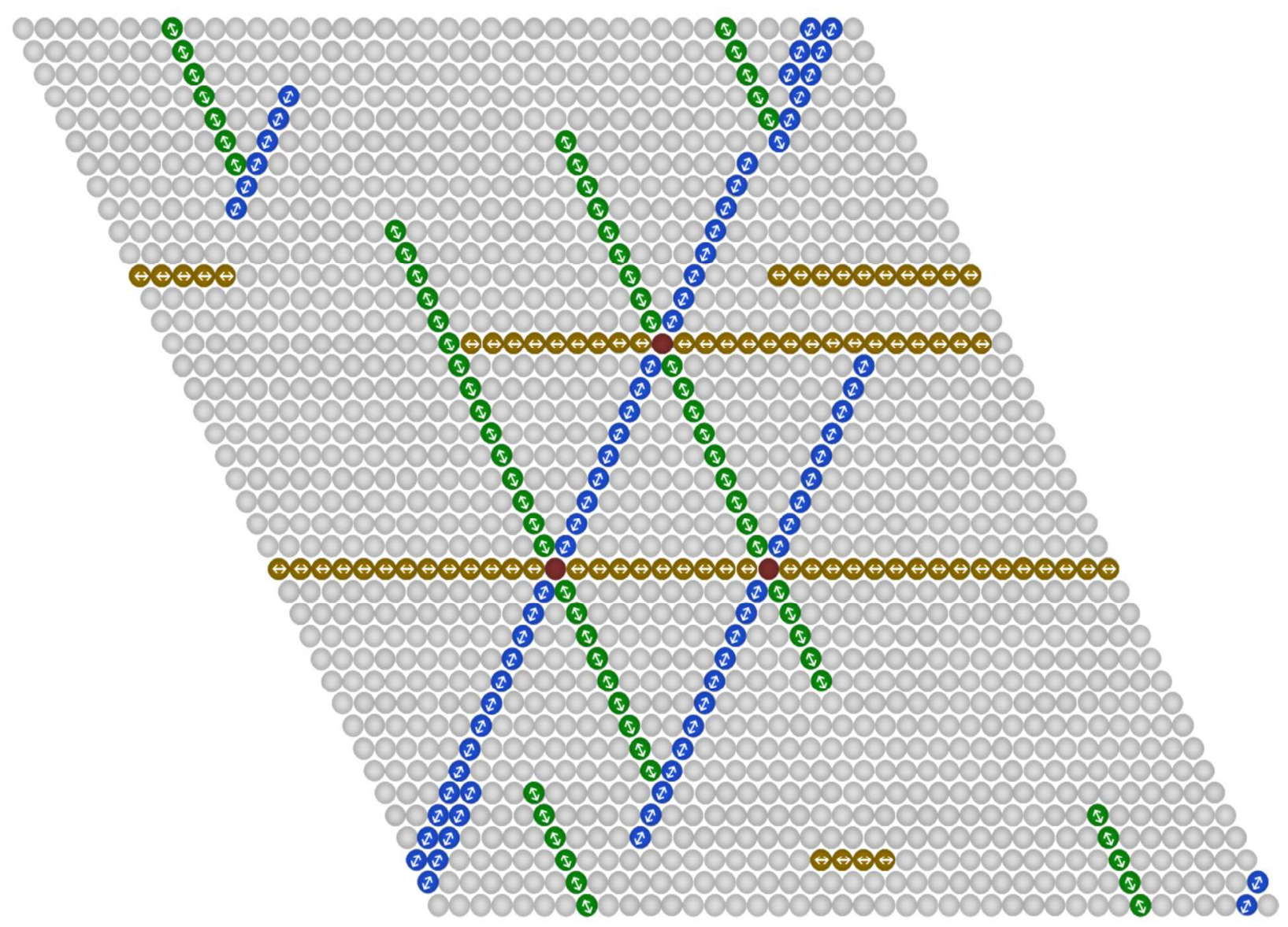


Figure 1: Schematic diagram of a $40 \times 40$ triangular lattice with periodic boundary conditions for a coverage $\theta \approx 0.12$. A typical elementary circuit unit $\mathrm{UCE}_{10}$ is shown. The blue, green and yellow circles represent monomeric units aligned along the three lattice directions, as shown by the arrows. The gray circles are the empty sites in the lattice and the red circles represent the fixed particles taking the role of nucleation sites.

We use a lattice gas model, where the surface is represented by an array of $N=L \times L$ sites in a triangular lattice arrangement (see Figure 1), analogous to the arrangement of surface atoms on $\mathrm{Au}(111)$, where $\mathrm{L}$ is the linear size of the array. Periodic boundary conditions are imposed to represent an infinitely large system. Each site can be occupied by a monomeric unit of the molecular wire, where the monomeric unit is also given a value of an alignment parameter corresponding to one of the three surface directions, analogous to the (Au-PDI) monomeric unit. Note here the first difference between the simplified model and the experimental system. In the model, each monomeric unit occupies a single site in the triangular lattice while in the experimental case each (Au-PDI) unit extends linearly thus occupying four unit cells of the Au(111) surface. ${ }^{13}$ The lattice direction of the monomers in the model is manifested by attractive interaction energies in one of the three lattice directions, as given by the alignment parameter. The direction of the interaction energy is shown by a double arrow in the representation of the monomeric units in Figure 1. As will be seen later, under certain circumstances, this linear interaction can induce the self-alignment of the monomers into chains on the surface, similar to the experiment case, when the interaction energies are similar to those in the experimental system. It was seen for the (Au-PDI) system that low coordination sites, such as step edges, dislocations, kinks or island edges on the surface can act as nucleation sites from which the molecular wires grow. It was already suggested in the first experimental report of this system that this fact could be exploited by patterning the surface with defects (low-coordination sites) to act as nodal points for the self-assembly of nano-architectures. ${ }^{13}$ While there is not yet direct experimental evidence of this, a recent conductivity study of a mica surface covered by gold nanoparticles shows that the latter are electrically linked upon adsorption of PDI by inducing the formation of $(A u-P D I)_{n}$ wires linking them. ${ }^{14}$ We further elaborate here on this idea by arbitrarily postulating an initial structure of the particles locked in place on the triangular lattice that play the role of nucleation sites and interact with the 
mobile monomeric units in any of the three lattice directions, analogous to what was observed for gold islands on the $\mathrm{Au}(111)$ surface. Figure 2 shows an example of the experimental case obtained in our laboratory, imaged by scanning tunneling microscopy, in which gold islands connected by molecular wires are seen in the image.

In the following, fixed, low-coordination growth nucleation sites will be referred to as "nodes" or "nodal points". As will be seen later, the nodes have a remarkable influence on the resulting self-assembled structures. In our model the interaction of nodal points with AuPDI monomers is considered to be

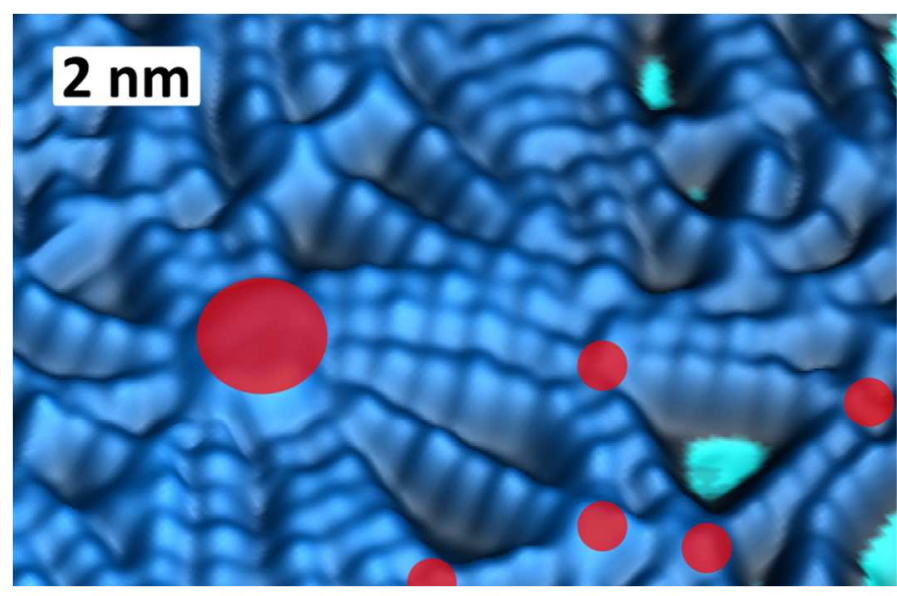

Figure 2. STM image of (Au-PDI) wires on Au(111). Nodal points where the (Au-PDI) $)_{n}$ wires originate from are emphasized by red circles. Imaging conditions: $I_{t}=206$ attractive and to occur only in the $p A, v_{b}=-2 \mathrm{~V}$.

direction of the axis determined by alignment parameter of the monomer, (see Figure 1). Then, the adsorbed phase, in the canonical ensemble (at a fixed coverage), is characterized by the following Hamiltonian:

$$
\begin{aligned}
H=-w \sum_{\langle i, j\rangle} \operatorname{int}\left\{\left|\vec{r}_{i j} \cdot \vec{s}_{j}\right|\left|\vec{r}_{j i} \cdot \vec{s}_{i}\right|\left(1-c_{i}\right)\left(1-c_{j}\right)\right\} \\
\quad-w \sum_{\langle i, j\rangle}\left[\operatorname{int}\left\{\left|\vec{r}_{i j} \cdot \vec{s}_{j}\right|\left(1-c_{j}\right) c_{i}\right\}+\operatorname{int}\left\{\left|\vec{r}_{j i} \cdot \vec{s}_{i}\right|\left(1-c_{i}\right) c_{j}\right\}\right]
\end{aligned}
$$

Here, the sums are over pairs $\langle i, j\rangle$ of nearest-neighbor (NN) sites; the occupation of one site by the Au-PDI monomer is described by the vector $\vec{s}_{i}=\left\{0, \vec{x}_{k}\right\}$, which takes the value " 0 " if the site is empty, and $\vec{x}_{\mathrm{k}}$ it is it occupied, giving as well the orientation of the monomer in one of the " $k$ " directions of the lattice; $\vec{r}_{i j}$ is the vector connecting site " $i$ " with site " $j$ "; the node occupation variable is given by, $c_{i}=0,1$, where $c_{i}$ takes the value " 0 " when the site does not have a nodal point; $w$ represents the lateral interaction between two adsorbed monomers 
located in two NN sites or the lateral interaction between a monomer and a NN node. In this model the interaction between nodes is not considered, since they form a regular array and are far apart from each other. A study with a more general model including this and other interaction terms is in progress. Thus, the first term on the right-hand side of eq.(1) represents the contribution to the Hamiltonian corresponding to particles located at sites $\mathrm{i}$ and $\mathrm{j}$, which are nearest neighbors; sites may or may not be occupied by monomers which have orientations given by $\vec{s}_{i}$ and $\vec{s}_{j}$; monomers contribute with energy $w$, if and only if both are oriented in the direction of the vector connecting the two sites $\vec{r}_{i j}$ or $\vec{r}_{j i}$; the final contribution is 0 or 1 , due to the function "int", which take the integer part of this factor. Finally, the factor $\left(1-c_{i}\right)\left(1-c_{j}\right)$ avoids the presence of nodal points at both "i" and "j" sites.

The second term represents the contribution to the Hamiltonian due to a monomer located at site $j(i)$ and a nodal point located at site $i(j)$. Note that, in the absence of nodes the model is the well-known Rigid-Rod Model, which has been extensively studied by others. For more details on the self-assembled rigid rod model, see ref. ${ }^{17}$ and references therein.

\section{Monte Carlo Method}

The thermodynamic properties of the present model have been investigated by means of a standard-importance-sampling Monte Carlo method in the canonical ensemble. As mentioned above, the simulation lattice is a triangular array of $N=L \times L$ sites with conventional periodic boundary conditions. Thermodynamic equilibrium is obtained by following the Kawasaki dynamic. ${ }^{18}$ As an initial configuration, a fixed number of nodes can be located at random or in a regular array (in what follows, only regular arrays can be considered); then, a fixed number of adsorbed monomer units is distributed on the empty surface sites, to reach a predefined coverage $\theta$, defined as the ratio between the number of adsorbed monomers and the total number of surface sites $\mathrm{N}$ ). The location and orientation of the monomers are taken at random. Then the system is allowed to reorganize itself based on the following rules. First, one of two possible events, migration or rotation, are chosen at random. For a migration event, an occupied site and an empty site are selected randomly, establishing its coordinates; they are not necessarily NN sites. Then, the difference between the energies of the final and initial 
states, $\Delta E=E_{f}-E_{i}$, is calculated; a random number $\xi$ uniformly distributed in the interval $(0 \leq \xi \leq$ $1)$ is chosen, and compared with a probability $P=\min [1, \exp (-\beta \Delta E)]$ where is $\beta=1 / k_{B} T\left(k_{B}\right.$ is the Boltzmann constant); an exchange between the occupation of the sites is carried out if $P>$ $\xi$, i.e.: if the probability $P$ is larger than the random number $\xi$.

For monomer rotation, the rotational state of the selected monomer is changed following the same procedure described above. A final rotation state is considered, and the energy of the monomer is calculated. Finally the energy change between the initial and final rotated states is computed. The new rotated state is accepted after considering the comparison between a random number and the probability as described above.

A Monte Carlo step (MCs) is defined as $N=L \times L$ attempts to change the state of occupation and rotation of a surface site. Typically, the equilibrium state is reached after $1 \times 10^{5} \mathrm{MCs}$, as will be shown later. In order to simplify the comparison of interaction energies, we will assume a temperature of $298 \mathrm{~K}$. However, it should be noted that, given the $\Delta E / k_{B} T$ term in the probability equation, when discussing changes in energy, this would be equivalent to inverse changes in temperature. Note that a dimensionless parameter could have been used instead to make the model more general. In fact, this dimensionless parameter can still be obtained by multiplying the stated energies throughout the manuscript by $k_{B} * 298 \mathrm{~K}$ if a more general term is needed.

\section{Results and Discussion:}

\section{a. Building an Elementary Circuit Unit (ECU).}

We start by defining a lattice of size $40 \times 40$ sites. To analyze the nano-scale selfassembly of molecular wires directed by nodal points, we define an "elementary circuit unit" (ECU) as a geometrical figure given by a regular arrangement of nodal points located at the vertices of such figure, i.e. for a triangular lattice, it can be a triangle (or a square or rectangle for a square lattice, etc.). That is an arbitrary definition, but it is necessary in order to derive the equilibrium properties of the system determined by the location of the nodal points. For example, a triangle in which the nodes are ten lattice units apart will be designated $\mathrm{ECU}_{10}$. 
Figure 1 shows an example of a $40 \times 40$ triangular lattice with $\mathrm{ECU}_{10}$ and monomers on the surface aligned in the three different directions denoted by different colors and arrows. The gray circles represent empty sites and the red circles are the fixed particles that act as nucleation sites.

Let us define the assembly probability $\mathrm{P}_{\mathrm{A}}$ as the number of simulations that result in the formation of an ECU divided by the total number of simulations for a given coverage. Here the coverage $\theta$ is defined as the total number of monomers plus nodal points divided by the total number of lattice sites. In Figure 3, the assembly probability $\mathrm{P}_{\mathrm{A}}$ of the $\mathrm{ECU}_{10}$ is plotted as a function of time (in MC steps) for various coverages using a lattice with dimension $L=40$. It can be seen in this plot that the system has already reached equilibrium after $10^{5} \mathrm{MCs}$. We have included a plot in the supporting information (Fig. S1) including times up to $10^{7} \mathrm{MCs}$ to show that there is no significant change in the value of $P_{A}$ for $t>10^{5} \mathrm{MCs}$. This time is substantially less than was used to stabilize previously reported self-assembled rigid rod (SARR) phases, which may be three orders of magnitude greater; see ref. ${ }^{17}$ and references therein. In the case described here however, the presence of nucleation sites contributes to reaching the thermodynamic equilibrium state significantly faster. It is worth here paying close attention to the effect of the coverage in the assembly probability. $P_{A}$ reaches a maximum in the range 0.142-0.299. For coverages lower and higher than this range the probability decreases. For the cases above this range, $\mathrm{P}_{\mathrm{A}}$ at equilibrium decreases in a smoother way and this decrease is likely due to crowding effects and the fact that, at higher coverages, large domains with a single monomer orientation will be formed hampering the formation of the ECU which requires the three wires in different orientations to form the structure. For lower coverages there is sharp change in behavior close to the minimum number of monomers necessary to form the elementary circuit unit $\left(\theta_{\min }=0.017\right)$. These are the cases of coverage 0.026 and 0.029 . The reason for the sharp change close to the minimum number of monomers to make the ECU is related to the fact that if the wire starts to grow from the nodal point in the wrong direction (not towards the other nodes) then it is hard for the system to correct itself and detach monomers to contribute toward the ECU at a fast enough rate. It is in fact more likely to form 
the ECU when there is a significant excess of monomers, note that a $\mathrm{P}_{\mathrm{A}}$ close to unity is obtained only when the coverage is four times the minimum coverage. Note as well that for coverages between $\theta_{\min }$ and $4 \theta_{\min }$, it takes longer to reach a thermodynamic equilibrium, as seen in the cases of $\theta=0.026$ and 0.029 .

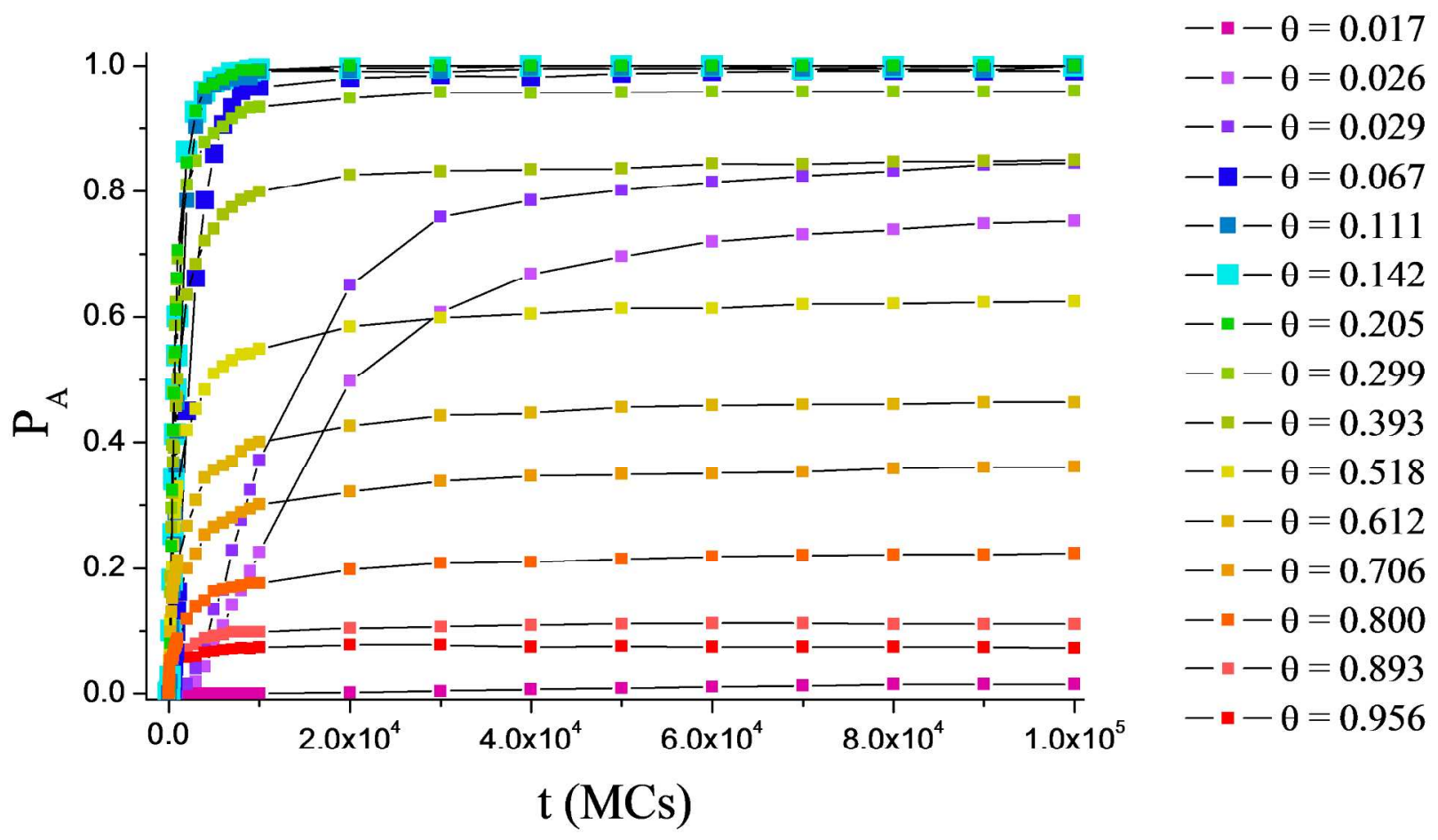

Fig. 3. Assembly probability $\left(P_{A}\right)$ of an ECUas a function of time (MCs) for a lattice of size $L=40$, with a nodal distance " $m$ " = 10 and an interaction " $w$ " = $124 \mathrm{~kJ} / \mathrm{mol}$ for various initial monomer coverages.

The equilibrium assembly probability as a function of coverage is shown in Fig. 4 for different values of lateral interaction energies.. It can be seen that, for the stronger interactions $(124 \mathrm{~kJ} / \mathrm{mol}$ and even for $24.79 \mathrm{~kJ} / \mathrm{mol}$ ), there is a range of coverages ( $\theta=0.1$ to 0.2 ) for which the probability of assembling the ECU is 1 . In the case shown here, for an $E_{10}$, the minimum number of monomers necessary to form an ECU is $\theta=0.017$. However, Fig. 4a shows that for this coverage, even if the number of monomers present is sufficient, the probability of actually forming the structure is close to 0 . For coverages larger than $\theta=0.2$, there is a linear decrease in the assembly probability reaching $P_{A}=0$ for $\theta=1$. To aid in visualizing the coverage effect on ECU formation, we have included snapshots of the surface as a function of coverage in Figure $4 \mathrm{~b}$. The assembly probability drops significantly for lower 
interaction energies; however the plots show a similar shape with maxima always at $\theta<0.5$. This means that, as the interaction energy decreases (or the temperature increases) the formation of self-assembled structures decreases due to the known reversibility of this process, tending to the formation of an isotropic phase. ${ }^{17}$

Some basic guiding principles can be obtained from the above discussion when aiming at the self-assembly of an ECU: i) When the exact number of monomeric units to form the ECU is present, the probability of actually assembling the ECU is virtually zero, ii) there is an optimum range of coverages where the probability of assembling the ECU is maximum and iii) the circuit is not formed at high coverages, most likely due to "crowding" effects.

It is worth emphasizing again at this point that the complexity of the real (PDI-Au $)_{n}$ system is not reflected in this simple model. However, the simulated assembly does agree with the main features of the experimental work on the (PDI-Au)n system. For example, while in this model monomers are allowed to jump to non-NN sites, it is likely that NN diffusion plays an important role in the assembly. While it is intuitive to think that diffusion is the only possibility in the experimental case, the experimental results at high coverages seem to indicate that this is not necessarily the case. Note that experiments at high coverages do show well-ordered domains (see figure 2 of ref. $^{19}$ ) similar to the ones found in the present work (high coverages in figure $4 b$ of this work). We do acknowledge that diffusion could lead to similar results (with a much higher computational cost). Another difference is that in the experimental case monomers are anisotropic occupying a line of four sites of the $\mathrm{Au}(111)$ lattice while in the model they occupy just one site of the lattice. This simplification is compensated by the fact that, in the model, the anisotropy is in fact present in sense that each monomer has a vector variable defining the anisotropic interaction. Finally, while in the simulations we restrict the growth of the wires to the three main lattice directions, there have been cases in the experimental system in which some flexibility is observed in the wires, especially near step edges. $^{13}$ 

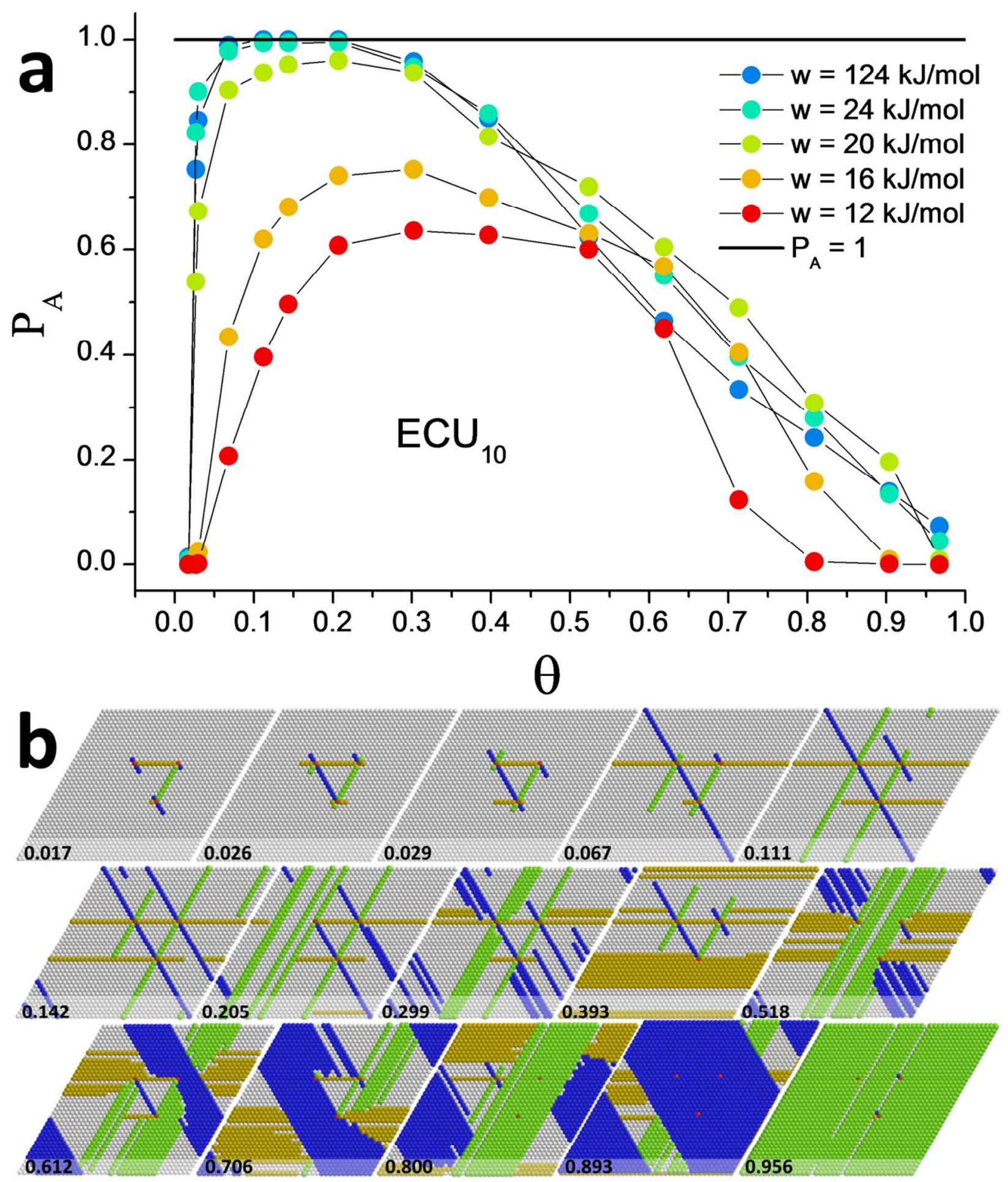

Fig. 4: a) Assembly probability $\mathrm{P}_{\mathrm{A}}$ as a function of coverage for different lateral interactions $w$ for an ECU of size $m$ $=10$. b) snapshots of the surface corresponding to the different coverages in the plot for $\mathrm{w}=124 \mathrm{~kJ} / \mathrm{mol}$, an energy close to that which was calculated for the experimental case of (PDI-Au $)_{n}$ on $A u(111) .{ }^{16}$ The three different colors 
(blue, green and yellow) represent the three different alignments that monomers have with the lattice. This also helps visualizing the formation of the wires by color-coding the monomers that form them.

In order to analyze the influence of the size of the ECU on the assembly probability, the length of the sides of the equilateral triangle and the number of building blocks to be self-assembled into the circuit were modified. The simulated experiment was performed using $\mathrm{w}=124 \mathrm{~kJ} / \mathrm{mol}$ and the time, $\mathrm{t}=10^{6} \mathrm{MCs}$, averaged over 1000 samples. The interaction energy was chosen to be close to a previously reported value calculated using ab-initio methods, including van der Waals interactions, for the formation of a link between two Au-PDI units starting from the noninteracting upright orientation of the monomeric units, with one of the units being fixed to a low coordination site, in that case a step edge. ${ }^{16}$

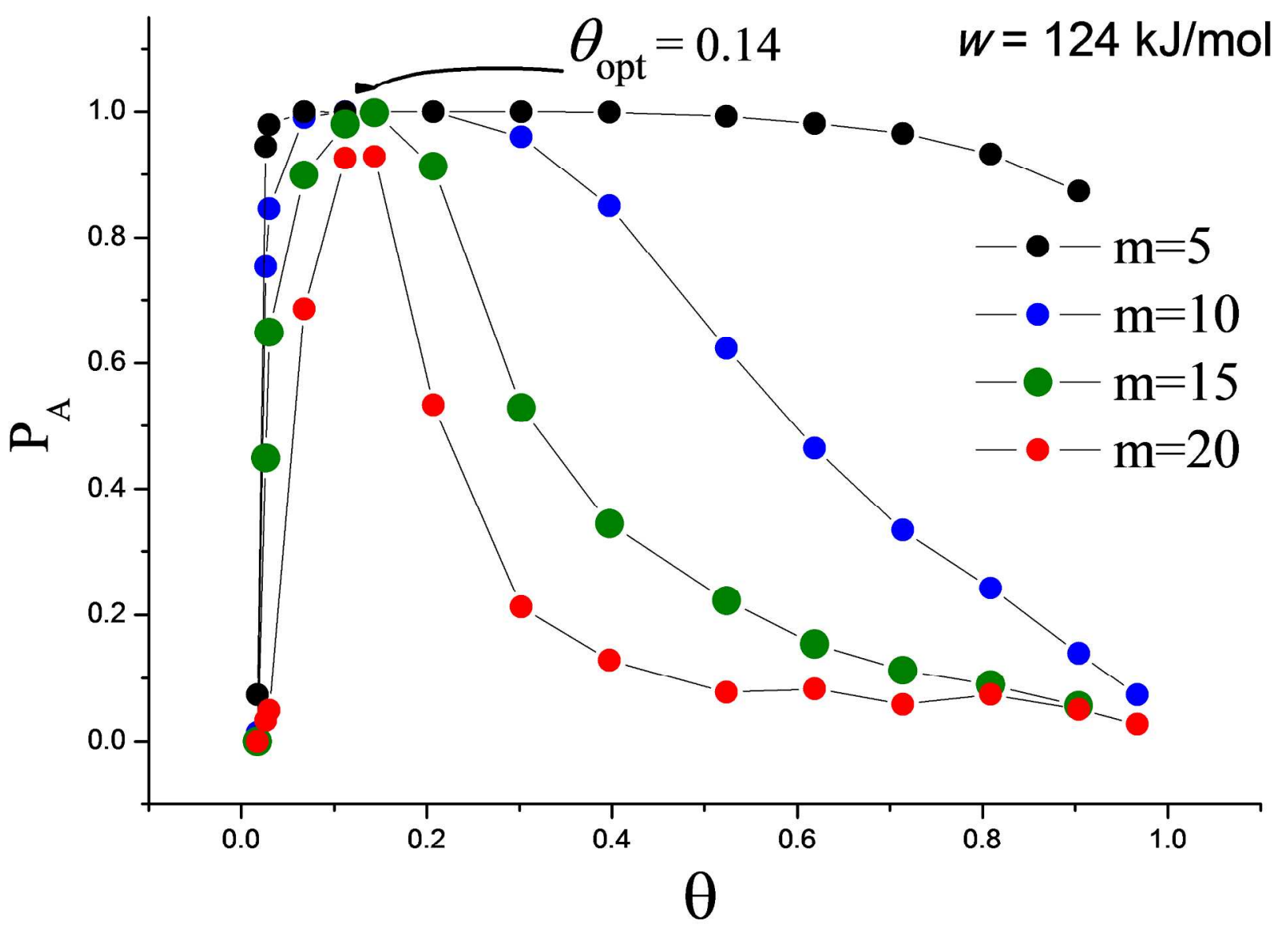

Fig. 5: The assembly probability of $\mathrm{ECU}_{\mathrm{m}}$ as a function of the coverage, for different values of separation between the nucleation sites, $m=5,10,15$ and 20 . Here $L=40, t=10^{6}$ MCS and averaging over 1000 experiences, for an interaction energy of $124 \mathrm{~kJ} / \mathrm{mol}$. 
In Figure 5, the assembly probabilities $\mathrm{P}_{\mathrm{A}}$ of the ECUs are plotted as a function of coverage for $\mathrm{w}$ $=124 \mathrm{~kJ} / \mathrm{mol}$ and for different separations between nucleation sites, $\mathrm{m}(\mathrm{m}=5,10,15$ and 20) units of the ECU. Interestingly, while the region of maximum probability decreases as the separation increases, the maximum of the curves corresponds to a common coverage, with a value of $\theta_{\mathrm{opt}}=0.14 \mathrm{ML}$, which is the optimum coverage. This means that the assembly probability of the ECU has the same $\theta_{\text {opt }}$ for a given system and interaction energy, regardless of the separation distance between the nodal points. It is important to note that the region of maximum probability becomes increasingly narrower as the separation distance increases. The spacing between nucleation sites and coverage of monomeric units is then critical when carrying out a "bottom up" synthesis of molecular self-assemblies, to obtain a given architecture. This is due to the fact that the range of useful coverages decreases as the separation increases. At a certain separation length, this is also accompanied by a decrease in the probability of obtaining the desired architecture, as in the case of $m=20$ in Fig. 5, where the probability of assembly at $\theta_{\text {opt }}$ decreases to $P_{A}=0.93$. Careful analysis of the simulated structures around the optimum coverage of 0.14 reveals that, at these coverages, the wires that grow from the nodal points span across the whole lattice. This is the case for example of the snapshot $\theta=0.142$ in figure $4 \mathrm{~b}$. It becomes clear when doing the math, that this is actually the coverage at which all the lattice sites aligned with the nodal points are covered by monomers.

\section{b. Circuit Assembly.}

In this section, the assembly of a "circuit", defined as a network of ECUs, will be discussed. Note that the goal of the present work is to introduce the proposed methodology for a simple case study making use of the previously defined ECU, without getting into more complex architectures found in a conventional electronic circuit. The circuits to which we refer here are simply controlled electrical connections between two sides of a surface, representing two electrodes for example, making use of the self-assembly of molecular wires guided by arrangement of nucleation sites (nodal points) on the surface. Despite its simplicity, it is of great interest to analyze this process so we can define the fundamental concepts that will aid 
the design of complex self-assemblies in the future. For example, in recent work by Kestell and coworkers, it was shown that $(A u-P D I)_{n}$ chains, which connect islands of gold atoms on a mica substrate, are good electrical conductors. ${ }^{14}$ Furthermore, it was also shown that the (Au-PDI) $n$ junctions can be switched off when they interact with certain molecules, such as $\mathrm{CO}$, which has important implications for different applications since the interconnecting of molecules can be considered as molecular "switches" that respond to chemical changes in the environment, such as the presence of a specific gas. ${ }^{19}$ These features are of particular interest from the practical point of view, as these switches could be used as very sensitive gas detectors.

While in the previous section, the formation of the $\mathrm{ECU}_{\mathrm{m}}$ was described as a function of the monomer coverage $\theta$, the interaction energy " $w$ " and the nucleation site separation " $m$ ", in this section, we describe the formation of a circuit consisting of a network of triangles (ECU) on a triangular lattice with periodic boundary conditions. The probability of the circuit assembly is analyzed as a function of $\theta$, aiming to find the optimum initial coverage required for circuit assembly.

The probability of circuit assembly, $\mathrm{P}_{\mathrm{A}}$, is defined in this case as the ratio between the number of ECU's present divided by the number of maximum possible ECU, for a lattice of size $L=160$, averaged over a large number of simulated experiments. Note that this definition for the circuit assembly is slightly different than that given previously for the single ECU formation.

Another important concept in designing self-assembled circuits is their connectivity, defined by its "percolation". A network of ECU's is said to percolate when there is a continuous conductive path of ECU's from one edge of the lattice to the opposite. It is very important to consider this continuity, and not only the assembly probability $\mathrm{P}_{\mathrm{A}}$, given the potential applications of these concepts to molecular electronics. There are three different ways in which the triangular ECU's can be connected, (1) through their edges, (2) through their vertices and (3) both through edges and vertices. For simplicity in the remainder of this analysis, we will focus on the case of triangular ECU's connected through their edges. To this end, ECU's of different sizes and lattice dimensions are analyzed. In Figure 5, it was shown that, for separations between nodes of up to $m=20$, the assembly probability approaches unity for the optimum monomer coverage, $\theta_{\text {opt }}=$ 
0.14 . Note that the analysis is for an interaction energy $w=124 \mathrm{~kJ} / \mathrm{mol}$, which is close to the value calculated for the experimental system. ${ }^{16,20}$ Larger interaction energies might allow reliable ECU assembly while smaller interaction energies might require shorter separations between nucleation sites. We will then focus on $m=20$ and $w=124 \mathrm{~kJ} / \mathrm{mol}$ for the remainder of this study. Given the experimentally measured distance of $\sim 1.1 \mathrm{~nm}$ between monomeric units, $m=20$ corresponds to a distance of $\sim 22 \mathrm{~nm}$ between nodal points. It should be noted that current e-beam nanolithography does allow surface patterning at this resolution so, in principle, these features, the nucleation sites, could be patterned on the surface to guide the self-assembly with currently existing technologies. Furthermore, the influence of the size of the system has been analyzed (data not shown), finding that $L=160$, is appropriate when considering the computational time required to obtain consistent results. With these features, a triangular lattice of $L=160$ with periodic boundary conditions and 64 nodal points is considered. The nodal points are located in such way that they form a network of ECU's superimposed on the triangular lattice. To analyze the dependence of $\mathrm{P}_{\mathrm{A}}$ in terms of coverage, a Monte Carlo simulation was carried out in the canonical assembly (constant coverage), and the kinetics of the process is described above. $1 \times 10^{6} \mathrm{MC}$ steps were used for these simulations and the reported $\mathrm{P}_{\mathrm{A}}$ value was obtained by averaging over 200 different samples.

Figure 6 shows the circuit assembly probability $\mathrm{P}_{\mathrm{A}}$ as a function of coverage. It can be seen that the probability is almost zero for low coverages, but then rises sharply to a peak at a coverage $\theta_{C}$, for which $P_{A}\left(\theta_{C}\right)=0.95$, (for this system $\theta_{C}=0.143$ ). For higher coverages, the curve decays smoothly. To determine the condition for which the ECU circuit is connected throughout the whole lattice, it is necessary to define the method by which they are connected. That will depend on whether the connection between ECU's is through vertices, edges, or both edges and vertices. As mentioned above, to simplify the analysis, here we consider a network of connections made through their edges. In this model, we can imagine a new lattice defined such that the center point of each triangle is a point in the new lattice. This results in a hexagonal lattice. See supporting figure S2 for schematics of a network of triangles connected by the edges. The percolation threshold for a hexagonal lattice has been previously studied and it can be exactly described by the following analytic equation: $T_{P}=1-2 \sin (\pi / 18)=0.6527036$. 
${ }^{21}$ This value is indicated as the solid horizontal line in Figure 6 . The intersection between the probability curve and the percolation threshold determines a coverage range $\mathrm{R}$ where the formation of a circuit that percolates through the lattice is possible and is bound by $\theta_{\min }$ and $\theta_{\text {max }}$. This reasoning can easily be extended to other ECUs and lattices with other connectivity, following the same procedure.

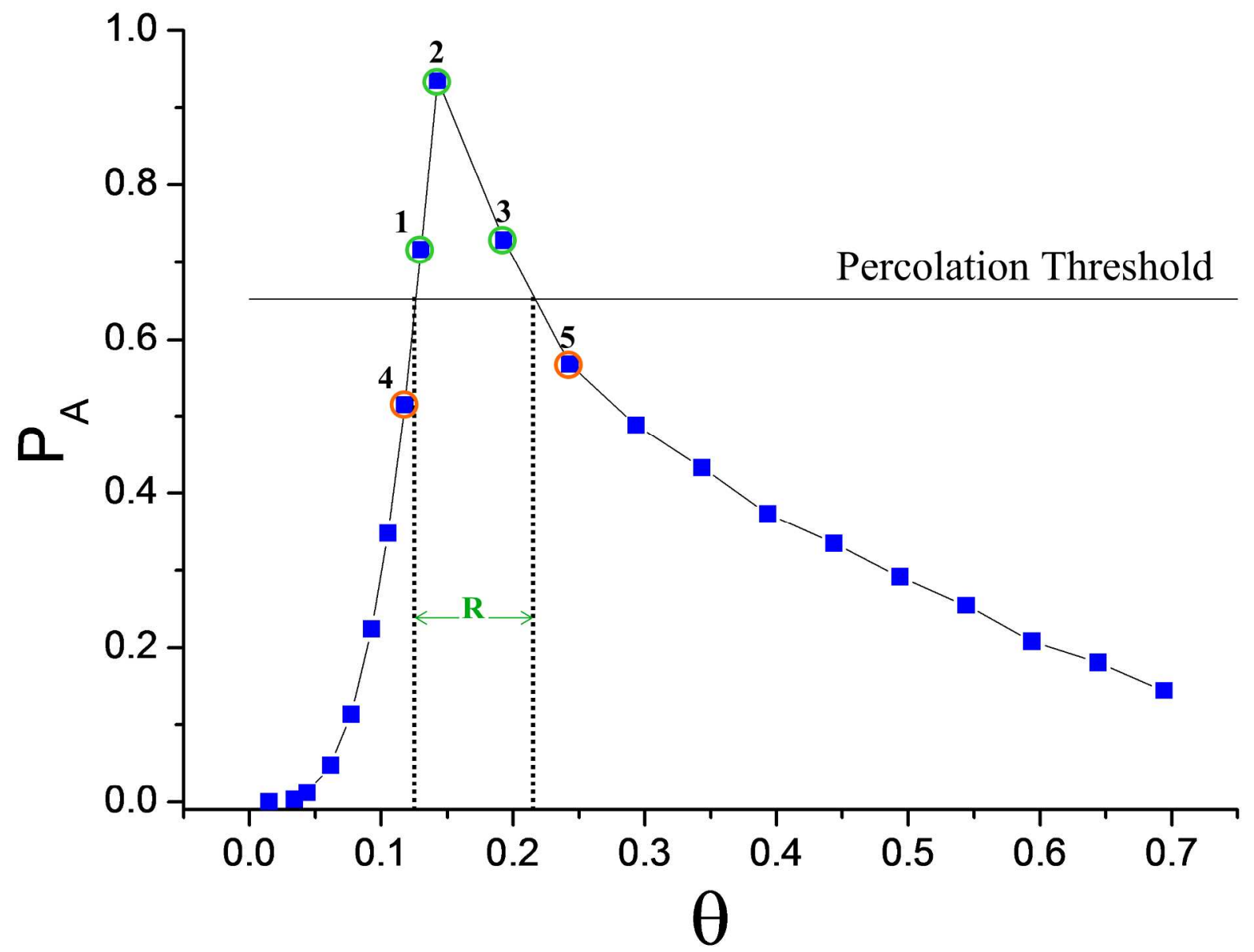

Fig. 6: Probability of circuit assembly $P_{A}$ as a function of coverage. $R$ is the range of coverages for which the ECUs form a percolated network. See figure 7 for snapshots of the surface at coverages below and above the percolation threshold.

Figure 7 shows snapshots of the simulated surfaces at the points 1-5 in figure 6. Points 1-3 are above the percolation threshold, and thus there is a path of ECU that connects the lattice from one side to the other. Points 4 and 5, which are below the percolation threshold, do not have a continuous path from side to side going through the ECUs, either due to the lack of monomeric units as is the case for point 4 or due to an excess of monomeric units that hampers the 
formation of sufficient ECUs, as is the case for point 5. Higher-resolution versions of each panel of figure 7 are included in the Supporting Information (see Figs. S3 to S7), where individual monomers forming the structure can be distinguished.

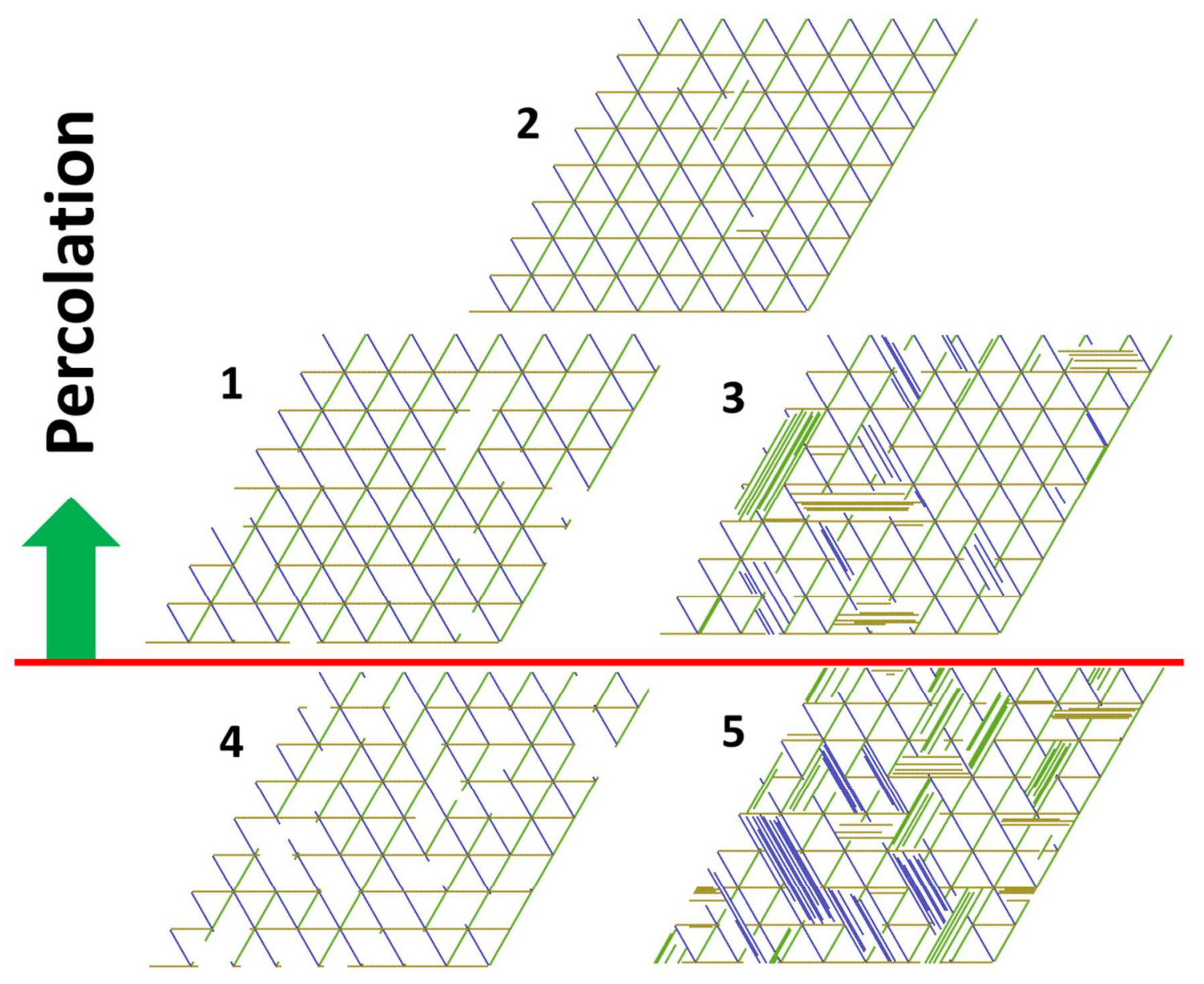

Fig. 7: Snapshots of simulations at points 1 though 5 emphasized in the plot in Fig. 6 . Points 1-3 are above the percolation threshold while point 4-5 are below the precolation threshold.

\section{Conclusions}

Simulated experiments on the self-assembly of molecular wires guided by the presence of nucleation units fixed on the surface were carried out using Monte Carlo methods using the lattice-gas model. This work is inspired by and compared to experimental data for the selfassembly of Au-PDI units on $\mathrm{Au}(111)$ into long (Au-PDI) $)_{n}$ oligomeric metal-organic wires. The experimental observation that nanowires are anchored to low-coordination sites such as surface dislocations, elbows of the herring-bone reconstruction and edges of gold islands is 
represented in the simulations by postulating fixed nucleation units on a triangular lattice at arbitrary locations and by defining attractive interactions with the mobile monomeric units. In order to facilitate the quantitative measurement of the self-assembly process, three nucleation sites at a distance " $m$ " unit cells apart were located on the surface to form an equilateral triangle and by defining an elementary circuit unit $\left(E C U_{m}\right)$ that is formed when wires selfassemble to connect these three nucleation sites. The probability of formation of the $\mathrm{ECU}_{\mathrm{m}}$ was then explored as a function of various parameters including the coverage of monomeric units, the distance " $m$ " between nucleation sites, the number of Monte Carlo steps, and the interaction energies. It was concluded that, for interaction energies on the order of those previously calculated for the experimental (Au-PDI) system ( $120 \mathrm{~kJ} / \mathrm{mol})$, the $\mathrm{ECU}_{\mathrm{m}}$ forms readily at $298 \mathrm{~K}$ when " $m$ " is small and the probability of ECU formation is optimum at a coverage $\theta=0.135 \mathrm{ML}$. Interaction energies lower than $24 \mathrm{~kJ} / \mathrm{mol}$ start to show a significant decrease in the probability of $\mathrm{ECU}_{10}$ formation at $298 \mathrm{~K}$, even at the optimum coverage of $\theta=$ $0.135 \mathrm{ML}$.

Adding another step in complexity, the percolation of a network of ECUs through the lattice was considered. It was found that combining the results from the simulations with the wellknown analytical expression for percolation thresholds, a range of coverages at which the network percolates from one side to the opposite of the lattice can be defined. This has important implications for the future design of guided self-assembled molecular electronics, since it allows the determination of the optimum values for a variety of design factors, such as the range of coverages of monomeric units that needs to be deposited on the surface to ensure electrical continuity and temperature at which the self-assembly process should occur.

\section{Acknowledgements}

Alejandro Boscoboinik acknowledges CONICET Argentina for doctoral fellowship. We thank Dr. J. Kestell and Dr. D. Stacchiola for useful discussions. This research used resources of the Center for Functional Nanomaterials, which is a U.S. DOE Office of Science User Facility, at Brookhaven National Laboratory under Contract No. DE-SC0012704. 


\section{References}

1. Cavin, R. K.; Lugli, P.; Zhirnov, V. V., Science and Engineering Beyond Moore's Law. Proc. IEEE 2012, 100, 1720-1749.

2. Feynman, R. P., There's Plenty of Room at the Bottom. Engineering and Science 1960, 5, 22-36.

3. Aviram, A.; Ratner, M. A., Molecular rectifiers. Chem. Phys. Lett. 1974, 29, 277-283.

4. Xu, B.; Tao, N. J., Measurement of Single-Molecule Resistance by Repeated Formation of Molecular Junctions. Science 2003, 301, 1221-1223.

5. Kiguchi, M.; Miura, S.; Hara, K.; Sawamura, M.; Murakoshi, K., Conductance of a single molecule anchored by an isocyanide substituent to gold electrodes. Appl. Phys. Lett. 2006, 89, 213104.

6. Smits, E. C. P.; Mathijssen, S. G. J.; van Hal, P. A.; Setayesh, S.; Geuns, T. C. T.; Mutsaers, K. A. H. A.; Cantatore, E.; Wondergem, H. J.; Werzer, O.; Resel, R.; Kemerink, M.; Kirchmeyer, S.; Muzafarov, A. M.; Ponomarenko, S. A.; de Boer, B.; Blom, P. W. M.; de Leeuw, D. M., Bottom-up organic integrated circuits. Nature 2008, 455, 956-959.

7. Crommie, M. F.; Lutz, C. P.; Eigler, D. M., Confinement of Electrons to Quantum Corrals on a Metal Surface. Science 1993, 262, 218-220.

8. Crommie, M. F.; Lutz, C. P.; Eigler, D. M., Imaging standing waves in a two-dimensional electron gas. Nature 1993, 363 (6429), 524-527.

9. $\quad \mathrm{Yu}, \mathrm{B} . ;$ 2006, 50, 536-544.

10. Barth, J. V., Fresh perspectives for surface coordination chemistry. Surf. Sci. 2009, 603, 15331541.

11. Barth, J. V., Molecular architectonic on metal surfaces. Annu. Rev. Phys. Chem. 2007, 58, 375-

407.

12. Whitesides, G. M.; Grzybowski, B., Self-Assembly at All Scales. Science 2002, 295, 2418-2421.

13. Boscoboinik, J. A.; Calaza, F. C.; Habeeb, Z.; Bennett, D. W.; Stacchiola, D. J.; Purino, M. A.; Tysoe, W. T., One-dimensional supramolecular surface structures: 1,4-diisocyanobenzene on Au(111) surfaces. Phys. Chem. Chem. Phys. 2010, 12, 11624-11629.

14. Kestell, J.; Abuflaha, R.; Boscoboinik, J. A.; Bai, Y.; Bennett, D. W.; Tysoe, W. T., Linking gold nanoparticles with conductive 1,4-phenylene diisocyanide-gold oligomers. Chem. Commun. 2013, 49, $1422-1424$.

15. Zhou, J.; Acharya, D.; Camillone, N.; Sutter, P.; White, M. G., Adsorption Structures and Electronic Properties of 1,4-Phenylene Diisocyanide on the Au(111) Surface. J. Phys. Chem. C 2011, 115, 21151-21160.

16. Garvey, M.; Kestell, J.; Abuflaha, R.; Bennett, D. W.; Henkelman, G.; Tysoe, W. T., Understanding and Controlling the 1,4-Phenylene Diisocyanide-Gold Oligomer Formation Pathways. J. Phys. Chem. C 2014, 118, 20899-20907.

17. López, L. G.; Linares, D. H.; Ramirez-Pastor, A. J.; Stariolo, D. A.; Cannas, S. A., Critical behavior of self-assembled rigid rods on two-dimensional lattices: Bethe-Peierls approximation and Monte Carlo simulations. J. Chem. Phys. 2013, 138, 234706.

18. Kawasaki, K., Diffusion Constants near the Critical Point for Time-Dependent Ising Models. I. Phys. Rev. 1966, 145, 224-230.

19. Kestell, J.; Boscoboinik, J. A.; Cheng, L.; Garvey, M.; Bennett, D. W.; Tysoe, W. T., Structural Changes in Self-Catalyzed Adsorption of Carbon Monoxide on 1,4-Phenylene Diisocyanide Modified Au(111). J. Phys. Chem. C 2015, 119, 18317-18325. 
20. Boscoboinik, J.; Kestell, J.; Garvey, M.; Weinert, M.; Tysoe, W. T., Creation of Low-Coordination Gold Sites on Au(111) Surface by 1,4-phenylene Diisocyanide Adsorption. Top. Catal. 2011, 54 (1-4), 2025.

21. Sykes, M. F.; Essam, J. W., Exact critical percolation probabilities for site and bond problems in two dimensions. J. Math. Phys. 1964, 5, 1117-1127. 


\section{Table of Contents Graphic}

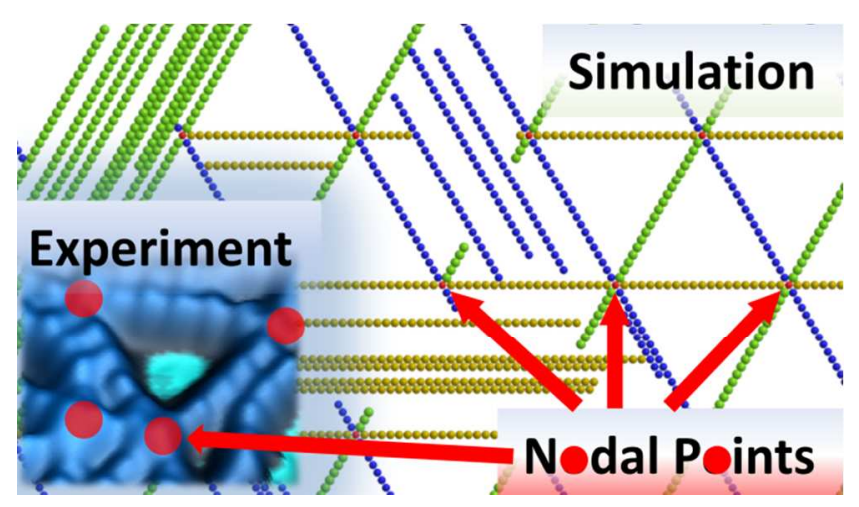

